\title{
Tcs evolution of CICC during Testing
}

\author{
D. Kaverin
}

All-Russian Scientific R\&D Cable Institute (JSC “VNIIKP”), Moscow, Russia

\begin{abstract}
ITER toroidal field coils in total by 6 different teams based on 5 different suppliers of $\mathrm{Nb}_{3} \mathrm{Sn}$ wires. Despite the fact that all conductors and superconducting wires were manufactured according to the same criteria, there were some differences in the manufacturing technologies of the components themselves, and in the result of the acceptance tests. Samples of some of the conductors passed acceptance tests, where the dependence of Tcs on the number of cycles of inputting the operating current into the samples when they were in a magnetic field was determined. The results of these tests showed that there are not only quantitative, but also qualitative differences in the $T c s(N)$ dependence. These differences are also observed within the same supplier. It would be useful to understand which parameters most strongly contribute to Tcs $(N)$ in order to possibly improve this characteristic. For this purpose, the article provides a statistical analysis of the test results of 49 samples of conductors. The results are being discussed.
\end{abstract}

\section{INTRODUCTION}

The conductors for the ITER toroidal field coils were manufactured by 6 different parties (suppliers), in an amount proportional to the in-kind contribution to the ITER project. All TF conductors were made on the basis of $\mathrm{Nb}_{3} \mathrm{Sn}$ strands, which were provided by one of 8 suppliers. The electrical and mechanical characteristics of superconducting wires can vary significantly from supplier to supplier [1], so the influence of superconducting wires is of key importance.

Despite the fact that the scheme, order and set of operations in the manufacture of conductors for each supplier are identical, there was still some difference in the technological modes and methods of their manufacture. In addition to some difference in the technology of manufacturing conductors, there was a sometimes significant difference in the characteristics of the materials used. Despite some difference both in the technology of manufacturing conductors and in the characteristics of the materials used, uniform acceptance criteria were applied to all conductors according to the quality system of the manufactured products. According to this system, the entire technological process of manufacturing conductors was monitored, starting from the incoming inspection of raw materials and ending with the final electromagnetic tests. While proof tests were performed on each conductor to characterize the design, electromagnetic tests were performed on only $25 \%$ of the total number of conductors. Final Electromagnetic Conductor tests are tests that simulate working load in a magnetic system (operating current is equal 68kA, background magnetic field is equal $10.78 \mathrm{~T}$ ). During the tests, the current was introduced into the samples cyclically from 0 to $68 \mathrm{kA}$ and vice versa. Acceptance test parameter $-\mathrm{T}_{\mathrm{cs}}$ after 1000 electromagnetic cycles. A typical dependence is $\mathrm{T}_{\mathrm{cs}}$ degradation, because $\mathrm{Nb}_{3} \mathrm{Sn}$ is a brittle material and is damaged during electromagnetic cycling with a corresponding decrease in the critical current density. Thus, the expected behavior of Tcs is its decrease with the number of test cycles. But at the same time, it can be seen that a completely different behavior is typical for one of the suppliers, namely, an increase in $\mathrm{T}_{\mathrm{cs}}$ and stabilization. Moreover, upon closer examination within suppliers, one can notice a fundamental difference in the behavior of $\mathrm{T}_{\mathrm{cs}}$ from conductor to conductor. This proves once again that technology can make a significant contribution to $\mathrm{T}_{\mathrm{cs}}$.

Due to the fact that each conductor had its own unique set of initial components and due to the deviations of technological modes from the nominal ones, the test results even within one supplier were fundamentally different. Considering the typical $\mathrm{T}_{\mathrm{cs}}$ dependencies between the first cycle and the considered one for each supplier, you can also see fundamentally different behavior during testing. Therefore, it is of interest to determine which parameters of conductors or characteristics of $\mathrm{Nb}_{3} \mathrm{Sn}$ wires make a significant contribution to $\mathrm{T}_{\mathrm{cs}}$ and to assess the degree of influence of each parameter separately. One way to determine these parameters is by statistical method.

Thus, the goal of the study can be formulated, which is as follows:

Determination of parameters that significantly contribute to $\mathrm{T}_{\mathrm{cs}}$;

Determination of the empirical equation connecting $\mathrm{T}_{\mathrm{cs}}$ and the found parameters;

On the basis of an empirical equation and statistical analysis, formulate a phenomenological model of the behavior of $\mathrm{T}_{\mathrm{cs}}$ conductors based on $\mathrm{Nb}_{3} \mathrm{Sn}$ stands.

\section{MATHERIALS, METHODS, ASSUMPTIONS}

Model of strain changing developed according to the principle of superposition - that is, as the sum of processes independent of each other. The model is phenomenological. Statistical analysis was carried out using more robust methods of parametric statistics. Statistical hypothesis testing was tested using various statistical tests. It is worth noting that the zone in which the maximum value (with an accuracy of $+/-0.1 \mathrm{~T}$ ) of the magnetic field is observed is a few millimeters over $450 \mathrm{~mm}$ of the conductor, so not all wires will fall into this zone - this can introduce a significant error in the analysis. As shown in [2], the Tcs of the same conductor can vary up to $+/-0.1 \mathrm{~K}$.

All data for statistical analysis are taken from the conductor database, developed specifically for quality control of supplied products within the procurement of conductors for the ITER magnetic system [3]. Obviously, the wires in one conductor differ from each other in the value of the critical current, but also in other parameters [4], therefore, for each 
of these wires, the curve $I=f(T, B, \varepsilon)$ will be different. In this work, we will assume that the strands have the same parameterization parameters, as described in [1], which are equal to the arithmetic mean of all superconducting wires in the cable.

\section{STRANDS STRAIN CHANGING DURING CONDUCTOR TEST}

We will consider three independent processes, namely: the change in the $\mathrm{T}_{\mathrm{cs}}$ of the conductor due to the training of the conductor, due to the change in strain during the test, and due to the rupture of the superconducting filaments of $\mathrm{Nb}_{3} \mathrm{Sn}$ wires as a result of their movement during the test. All three of these processes will be considered from a phenomenological point of view.

\section{A. Conductor Training}

As shown in [5, 6], as a result of the transition of the magnet to the normal state, each time it passes at different values of the current (the test temperature remains unchanged). This is associated, in a dignified way, with grinding wires to each other (micromovements during training), which decreases with each cycle, bringing less and less dissipated energy.

Based on the results of these works, it can be noted that the level of increase in the critical current can be characterized by some saturation with an increase in the number of input-output of current into the sample. Since, in general, the dependence of the critical current and critical temperature is uniquely characterized, it is possible to write the law of the critical temperature change on the number of tests as (1).

\begin{tabular}{|l|l|}
\hline$\Delta T_{c 1}(N)=a_{1} \cdot\left(1-e^{-b_{1} \cdot N}\right)$ & $(1)$ \\
\hline $\begin{array}{l}\Delta T_{c 1}(N) \text { - current sharing temperature changing on conductor training only; } N-\text { cycles number, } a_{1} \text { and } b_{1}- \\
\text { coefficients. }\end{array}$
\end{tabular}

\section{B. Filaments Cracking}

In the past, 2 independent works have been carried out to study the presence of breaks in superconducting filaments of strands after electromagnetic tests. In [7], studies were carried out on strands taken from a conductor, which showed a significant decrease in $\mathrm{T}_{\mathrm{cs}}$ during testing. In another work [8], similar studies were carried out on strands taken from another conductor, which, on the contrary, showed an increase in $\mathrm{T}_{\mathrm{cs}}$ during these tests. In both cases, significant breaks in the superconducting filaments in the strands were found, especially in the area near the conductor jacket, where the magnetic field is maximum. The quantitative results of both studies are comparable - that is, the specific number of breaks of superconducting filaments per unit length did not differ significantly. Here it is difficult to unambiguously judge the dependence of the change in $T_{c}$ on the number of breaks in superconducting filaments, since there is no experimental justification for this, but the following reasonable assumption can be made.

Filaments breaks only lead to a decrease in $\mathrm{T} c$; the greatest decrease in $\mathrm{T}_{\mathrm{c}}$ will be observed during the first few cycles, since there is the greatest movement of the wires, which means that the derivative of the function $\mathrm{T}_{c}(\mathrm{~N})$ must decrease continuously over the course of the test cycle; with a large amount of $\mathrm{N}$, a certain limit of the movement of the wires will come and the relaxation of the wires, the effect from the current output will not affect the decrease in Tc, therefore saturation will be observed. Based on these considerations, let us present the dependence of the change in $T_{c}(N)$ only on filaments breaks in the form (2).

$$
\Delta T_{c 2}(N)=a_{2} \cdot\left(e^{-b_{2} \cdot N}-1\right)
$$

$\Delta T_{c 2}(N)$ - current sharing temperature changing on conductor training only; $N$ - cycles number, $a_{2}$ and $b_{2}-$ coefficients.

Qualitative plots of $\mathrm{T}_{\mathrm{cs}}$ changes from training and from filament breaks are shown in Figures 1 and 2, respectively.

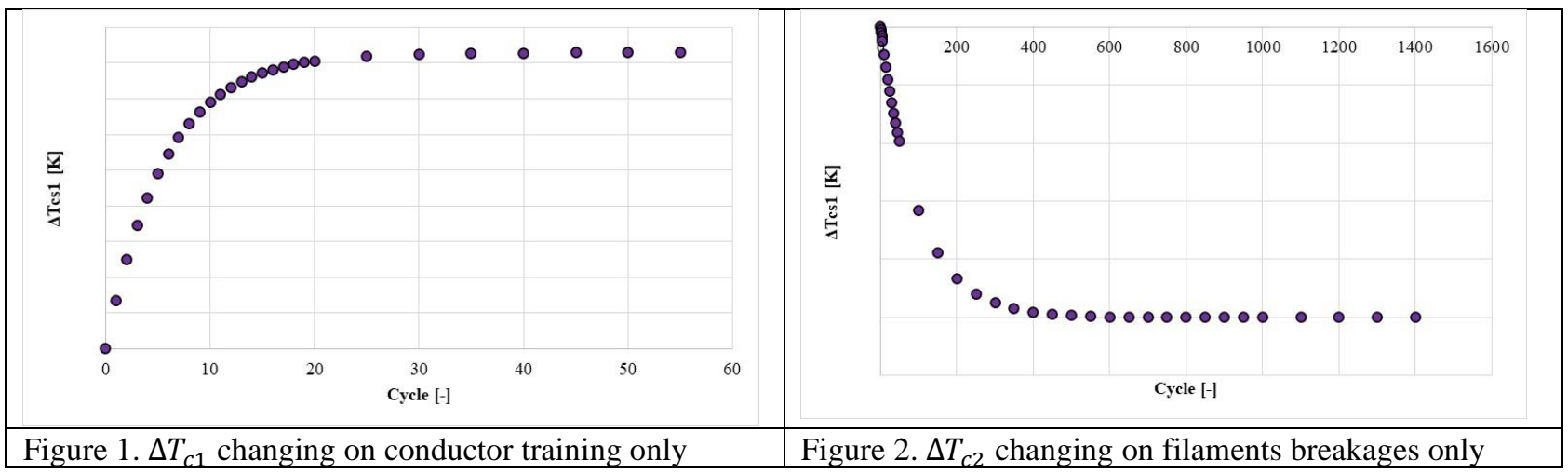


The relative elongation of wires after heat treatment, during cool-down and during testing can vary significantly. Based on the same considerations as for filaments breaks, strain will constantly decrease during testing due to relaxation. Indeed, the wires in the conductor support each other, including due to the frictional forces between the strands. When current is input or out, they move, which leads to a decrease in the interaction between the strands, reducing the strain. The magnitude of this change decreases with each cycle, but epsilon always decreases - the derivative is always negative. Obviously, saturation will also be observed. Thus, strain on the number of current input-output cycles will have the same form of dependence as (2). To plot the dependence of $T_{c}$ on the number of cycles, it is necessary to know the dependence of $T_{c}$ on strain. This dependence can be found from the data [1] for a current of 76A, which corresponds to an operating current of 68kA for the entire conductor for 900 superconducting strands. This dependence is approximated with a high accuracy in a parabola, and the dependence coefficients are known. A plot of a qualitative change in epsilon during testing and the dependence of the critical temperature of the strands at a current of 76A on different strain are shown in Figures 3 and 4.

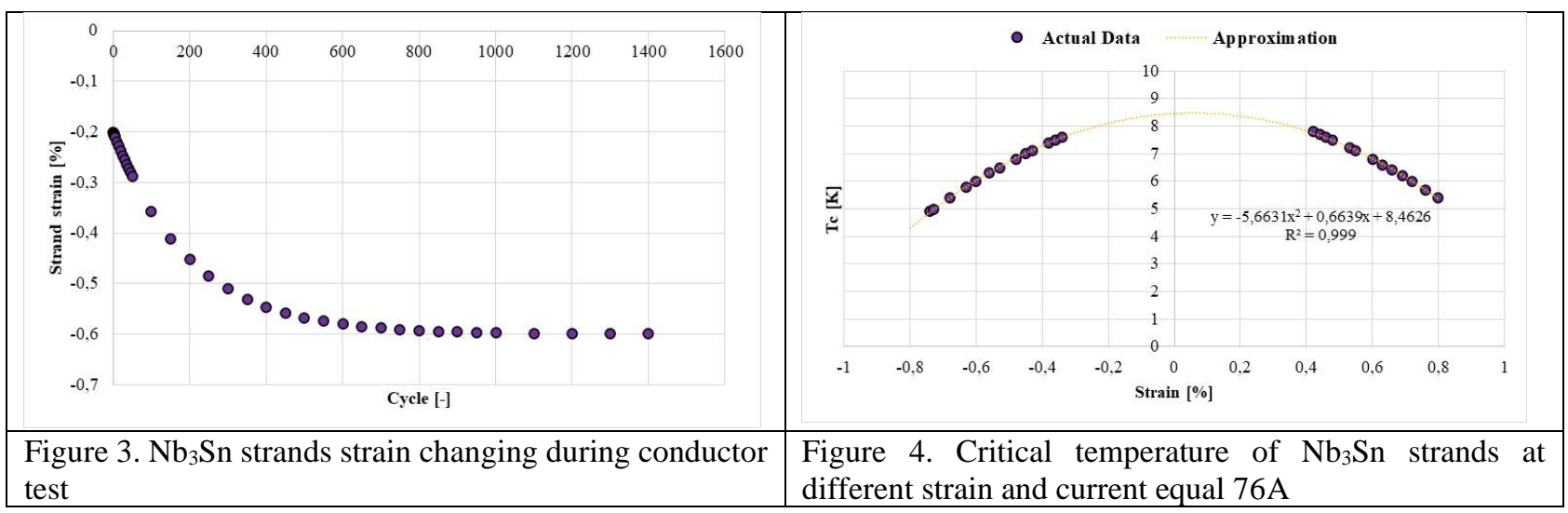

It should be noted that the initial value of strands strain can be completely different and depends on many factors, but in general they can be described as technological ones. These factors may be the force on the pay-off coils during cabling; the pulling force applying to cable during insertion into jacket; the degree of compression of the cable inside the conductor. All these parameters can significantly affect the initial strain of the strands, which ultimately leads to a qualitative change in the $\mathrm{T}_{\mathrm{cs}}$ of the conductor. To demonstrate the qualitative change in $\mathrm{T}_{\mathrm{cs}}$, Figure 5 shows possible variants of its change for different initial strain of strands, but with the same change in strands strain during the tests. As can be seen from the presented dependences, $\mathrm{T}_{\mathrm{cs}}$ can have completely different behavior - from an instant fall to an increase and saturation of $\mathrm{T}_{\mathrm{cs}}$ during tests.

\section{Tcs evolution model}

Let us find the total change in $\mathrm{T}_{\mathrm{cs}}$ during testing as the sum of changes in $\mathrm{T}_{\mathrm{cs}}$ for each of the three processes. Figure 6 shows the dependences of the change in $\mathrm{T}_{\mathrm{cs}}$ of conductors during the test, depending on the initial parameters.

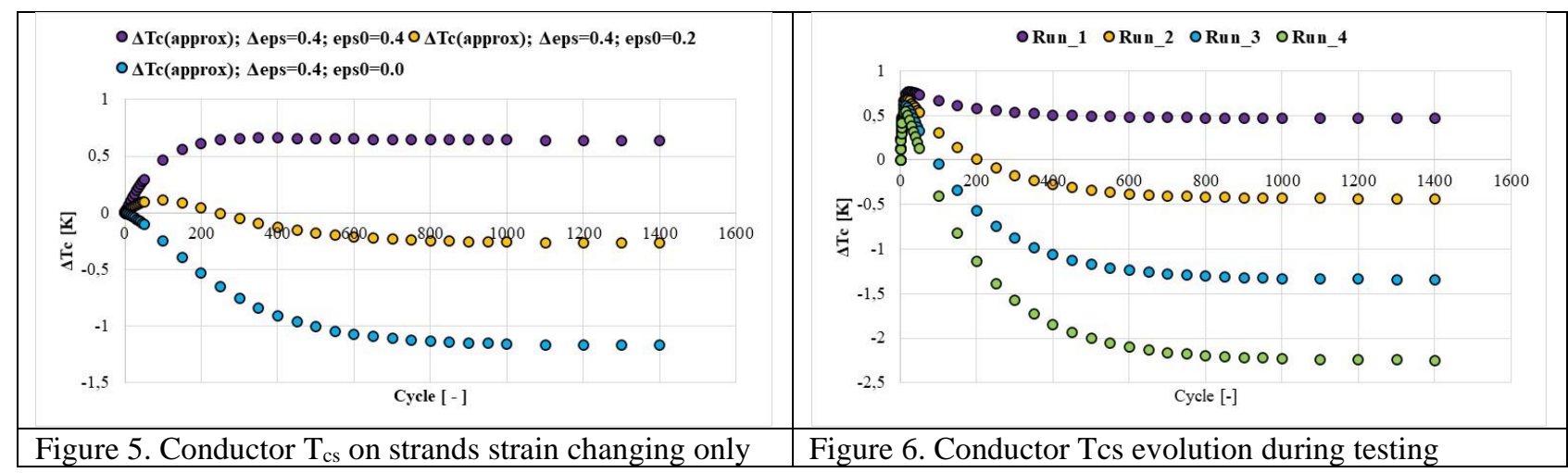

Thus, by changing the initial deformation of the strands, it is possible to achieve that the $\mathrm{T}_{\mathrm{cs}}$ of the conductors during the tests will increase with the number of cycles. This can be achieved due to the technology of manufacturing conductors, namely the higher tension of the superconducting strands during cabling and due to the greater compaction of the peripheral layer of the superconducting wires, thereby giving them greater mechanical stress.

\section{DISCUSSION}

It is necessary to conduct research for each separate group of conductors in order to understand the general pattern of $\mathrm{T}_{\mathrm{cs}}$ behavior. It is not clear how to determine the initial (before testing the conductor) strain in the strands. With an identical technology, it can be assumed that the original strain from each of the suppliers is the same, while the strain may differ 
from manufacturer to manufacturer. To generalize the model, it is necessary to conduct a detailed statistical analysis and determine what factors can influence the $\mathrm{T}_{\mathrm{cs}}$, although it is obvious that the mechanical characteristics of the wires make a key role in changing the $\mathrm{T}_{\mathrm{cs}}$, namely, their resistance to bending deformations and the initial strain value in the strands.

\section{CONCLUSION}

The paper proposes a new phenomenological model for the evolution of $\mathrm{T}_{\mathrm{cs}}$ during full-scale testing of conductors. It is shown that, under certain conditions, an increase in $\mathrm{T}_{\mathrm{cs}}$ is possible during tests, provided that the average strain of the strands is in the tensile zone before the start of the tests. One of the key factors in the behavior of $\mathrm{T}_{\mathrm{cs}}$ conductors is the resistance of superconducting filaments to damage and the initial stain of the strands in the composition of the conductor.

\section{ACKNOWLEDGEMENT}

This work was supported by Russian Science Foundation (project №19-79-00193).

\section{REFERENCES}

[1] A. Nijhuis et al., "Effect of axial and transverse loading on transport properties of ITER Nb3Sn strands", 2013 Supercond. Sci. Technol. 26084004

[2] D. Uglietti, R. Wesche, B. Stepanov, and P. Bruzzone, "Statistical Analysis of the Current-Sharing

Temperature Evolution in Nb Sn Cable-in-Conduit-Conductors for ITER", IEEE TRANSACTIONS ON APPLIED SUPERCONDUCTIVITY, VOL. 22, NO. 3, JUNE 2012, article 4802204

[3] Kazutaka Seo et al., "Status of the ITER Manufacturing Database for the Magnet System Procurement", June 2012IEEE Transactions on Applied Superconductivity 22(3):4203204-4203204, DOI:10.1109/TASC.2011.2175692

[4] A. Vostner et al., "Statistical analysis of the $\mathrm{Nb3Sn}$ strand production for the ITER $\mathrm{Nb}_{3} \mathrm{Sn}$ toroidal field coils", Supercond. Sci. Technol. 30 (2017) 045004 (14pp), DOI: https://doi.org/10.1088/1361-6668/aa5954

[5] G. Pasztor and C. Schmidt, "Dynamic stress effects in technical superconductors and the "training" problem of superconducting magnets", Journal of Applied Physics 49, 886 (1978); doi: 10.1063/1.324621

[6] H. Nomura, M.N.L. Sinclair and Y. Iwasa, "Acoustic emission in a composite copper NbTi conductor", CRYOGENICS. MAY 1980, pp. 283-289

[7] Carlos Sanabria et al., "Evidence that filament fracture occurs in an ITER toroidal field conductor after cyclic Lorentz force loading in SULTAN", Supercond. Sci. Technol. 25 (2012) 075007 (11pp), doi:10.1088/0953-2048/25/7/075007

[8] D Kaverin et al., "Analysis of $\mathrm{Nb}_{3} \mathrm{Sn}$ strand microstructure after full-size SULTAN test of ITER TF conductor sample", Physics Procedia 67, 914-919 